\title{
Real Schottky Uniformizations and Jacobians of May Surfaces
}

\author{
Rubén A. Hidalgo and Rubí E. Rodríguez
}

\begin{abstract}
Given a closed Riemann surface $R$ of genus $p \geq 2$ together with an anticonformal involution $\tau: R \rightarrow R$ with fixed points, we consider the group $K(R, \tau)$ consisting of the conformal and anticonformal automorphisms of $R$ which commute with $\tau$. It is a well known fact due to C. L. May that the order of $K(R, \tau)$ is at most $24(p-1)$ and that such an upper bound is attained for infinitely many, but not all, values of $p$. May also proved that for every genus $p \geq 2$ there are surfaces for which the order of $K(R, \tau)$ can be chosen to be $8 p$ and $8(p+1)$. These type of surfaces are called May surfaces.

In this note we construct real Schottky uniformizations of every May surface. In particular, the corresponding group $K(R, \tau)$ lifts to such an uniformization. With the help of these real Schottky uniformizations, we obtain (extended) symplectic representations of the groups $K(R, \tau)$. We study the families of principally polarized abelian varieties admitting the given group of automorphisms and compute the corresponding Riemann matrices, including those for the Jacobians of May surfaces.
\end{abstract}

\section{Introduction}

Let $R$ be a closed Riemann surface of genus $p \geq 2$ admitting an anticonformal involution $\tau: R \rightarrow R$ with fixed points. C. L. May (see [18]) proved that the upper bound $\nu(p)$ for the order of the group $K(R, \tau)$ of conformal and anticonformal automorphisms of $R$ which commute with $\tau$ is $24(p-1)$, and that it is attained for infinitely many, but not all, values of $p$. He also proved that $\nu(p) \geq 8(p+1)$ and $\nu(p) \geq 8 p$ if the quotient surface $R /\langle\tau\rangle$ is respectively orientable or non-orientable. The surfaces (of genus $p$ ) for which the order of $K(R, \tau)$ is exactly $8(p+1)$ or $8 p$ will be called May surfaces.

2000 Mathematics Subject Classification: Primary 30F40,14H15; Second. 14H40, 32G20. Keywords: Kleinian groups, Jacobians, automorphisms, abelian varieties. 
E. Bujalance (see [3]) gave an algebraic characterization of their corresponding groups and showed that the corresponding families of May surfaces are real analytic submanifolds of dimension one of the respective Teichmüller spaces. For every $p$, there is a special point in one of the families of May surfaces: the Accola-Maclachlan surface, given by the equation $w^{2}=z^{2 p+2}-1$. It has a conformal automorphism group of order $8(p+1)$, the largest possible that is realized for every $p$; it is also known (see [2]) that it admits three types of symmetries with fixed points, and we can see it is a May surface by considering precisely one of the three types. Its period matrix was computed in [4], using the real Riemann matrices approach.

Our aim here is the study of May surfaces from the point of view of real Schottky groups, that is, Schottky groups keeping invariant some Euclidean circle. Maskit proved (see [17]) that every compact Riemann surface $R$ with an anticonformal involution $\tau$ with fixed points may be uniformized by a real Schottky group so that the reflection on the corresponding Euclidean circle descends to the reflection $\tau$. We first construct real Schottky uniformizations for every May surface. In particular, the corresponding group $K(R, \tau)$ lifts to such a uniformization. This is given in sections 5 and 6 . In sections 7 and 8 we use these uniformizations to construct (extended) symplectic representations of the groups $K(R, \tau)$ and, in particular, to compute the families of principally polarized abelian varieties admitting the given group of automorphisms $K(R, \tau)$, which include the Jacobians of the uniformized May surfaces. The question of determining precisely which are period matrices for Jacobians (a Schottky problem question) will not be answered here, except of course for the Accola-Maclachlan surfaces.

\section{Symmetric Riemann surfaces: May surfaces}

A closed Riemann surface $R$ of genus $p \geq 2$ is called symmetric if it admits an anticonformal involution $\tau: R \rightarrow R$, called a symmetry of $R$. If such an involution has fixed points, then we call it a reflection on $R$.

We denote by $K(R, \tau)$ the total group of automorphisms (including orientation reversing transformations) of $R$ commuting with $\tau$. The quotient $S=R /\langle\tau\rangle$ is a compact (bordered) Klein surface of genus $g$ with $m$ bordered components, where $p=2 g+m-1$ ( $p$ is called the algebraic genus of $S$ ). The group $H(R, \tau)=K(R, \tau) /\langle\tau\rangle$ is isomorphic to the full group of automorphisms of $S$.

In the case $\tau$ is a reflection, it was shown by C.L. May in [18] that the order of $K(R, \tau)$ is bounded above by 24(p-1) (see also [11]) and provided examples for which $K(R, \tau)$ has order $8 p$ and $8(p+1)$ for every genus $p \geq 2$. In particular, the maximal order of $K(R, \tau)$ for reflections in genus $p \geq 2$ is at least $8(p+1)$ and at most $24(p-1)$. 
E. Bujalance in [3] obtained sufficient conditions for the group $K(R, \tau)$ to be of order $8(p+1)$, as follows.

Theorem ([3]) Let $R$ be a closed Riemann surface of genus $p \geq 2$ and $p \neq 5,11,29$ admitting a reflection $\tau: R \rightarrow R$ with $m \geq 1$ components of fixed points. If $K(R, \tau)$ has a subgroup $G$ of order $8(p+1)$ and containing $\tau$, then $G=K(R, \tau)$ and $G$ is isomorphic to the abstract group

$$
\begin{gathered}
K(p, r)=\left\langle x, y, z, w: x^{2}=y^{2}=z^{2}=w^{2}=1,\right. \\
\left.(w x)^{2}=(w y)^{2}=(w z)^{2}=(z y)^{2}=(x y)^{p+1}=1, z x z=y(x y)^{r}\right\rangle,
\end{gathered}
$$

for some $r$ such that $1 \leq r \leq p, r^{2} \equiv 1 \bmod (p+1)$ and for which $m=\operatorname{gcd}(p+1, r+1)$. Furthermore, the reflection $\tau$ corresponds to the generator $w$.

Conversely, for each group $K(p, r)$ as above and $m=\operatorname{gcd}(p+1, r+1)$, there exists a closed Riemann surface $R$ of genus $p$ with a reflection $\tau: R \rightarrow R$ having exactly $m$ components of fixed points so that $R / \tau$ is orientable and $K(R, \tau)=K(p, r)$.

In the above cases, $R / K(R, \tau)$ is the closed disc with four branch values in the boundary, one of order $(p+1)$ and the other three of order 2 .

Remarks. In the above theorem, the change of generators: $\widetilde{x}=x, \widetilde{y}=y$, $\widetilde{z}=y z, \widetilde{w}=w$ says that $K(p, r)=K(p, p+1-r)$, but when $\operatorname{gcd}(p+1, r+1) \neq$ $\operatorname{gcd}(p+1, p-r+2)$ the topological actions determined by the respective reflections $w$ are different.

For instance, there is a genus three Riemann surface admitting two commuting reflections $\tau_{1}$ and $\tau_{2}$, so that $\tau_{1}$ has exactly two components of fixed points and $\tau_{2}$ has exactly four. In this case, $K\left(R, \tau_{1}\right) \cong K(3,1)=K(3,3) \cong$ $K\left(R, \tau_{2}\right)$. But clearly $\left|K\left(R, \tau_{1}\right) \cap K\left(R, \tau_{2}\right)\right| \leq 16<32=|K(3,1)|$. In particular, $K\left(R, \tau_{1}\right) \neq K\left(R, \tau_{2}\right)$.

In general, the subgroup $K(R, \tau)^{+}=\langle w x, w y, w z\rangle$ of conformal automorphisms has presentations of the form

$$
K(R, \tau)^{+}=\left\langle a, b, c: a^{p+1}=b^{2}=c^{2}=(a b c)^{2}=1,(b c)^{2}=1, b a b=a^{r}\right\rangle
$$

and also

$$
K(R, \tau)^{+}=\left\langle a, b, c: a^{p+1}=b^{2}=c^{2}=1,(b c)^{2}=1, b a b=a^{r}, c a c=a^{-r}\right\rangle
$$

The first presentation is the adequate one for Unifweb [20], the second one shows that $K(R, \tau)^{+}$is isomorphic to the semi direct product of $D_{p+1}=$ $\langle a, b c\rangle$ and $\mathbb{Z} / 2 \mathbb{Z}=\langle b\rangle$.

A similar result, concerning the case that $K(R, \tau)$ has a subgroup of order $8 p$, is also obtained in Bujalance's paper [3]. In this case, $K(R, \tau) / \tau$ is isomorphic to the dihedral group of order $4 p$ and $R / \tau$ may be non-orientable. 


\section{Real Schottky uniformizations}

Let $p$ be some positive integer. Assume we have a collection of $2 p$ pairwise disjoint simple loops, say $\alpha_{1}, \ldots, \alpha_{p}, \widetilde{\alpha}_{1}, \ldots, \widetilde{\alpha}_{p}$, bounding a common region $\mathcal{D}$ of connectivity $2 p$. Let us also assume we have a collection of Moebius transformations $A_{1}, \ldots, A_{p}$ (necessarily loxodromic ones) so that $A_{j}\left(\alpha_{j}\right)=\widetilde{\alpha}_{j}$ and $A_{j}(\mathcal{D}) \cap \mathcal{D}=\emptyset$, for all $j=1, \ldots, p$.

The group $J$ generated by $A_{1}, \ldots, A_{p}$ turns out to be a Kleinian group, purely loxodromic, isomorphic to a free group of rank $p$ and with connected region of discontinuity; it is called a Schottky group of genus $p$ (for other equivalent definitions of Schottky groups see [16] and [5]). The domain $\mathcal{D}$ is called a standard fundamental domain for $J$ and the collection of loops $\alpha_{1}, \ldots, \alpha_{p}, \widetilde{\alpha}_{1}, \ldots, \widetilde{\alpha}_{p}$ is called a fundamental set of loops.

For any Schottky group $J, \Omega / J$ is a closed Riemann surface of genus $p$, where $\Omega$ denotes the region of discontinuity of $J$. The classical retrosection theorem states that if $X$ is a closed Riemann surface of genus $p$, then there is a Schottky group $J$ with region of discontinuity $\Omega$ and $P: \Omega \rightarrow X$ a normal covering map, with $J$ as covering group. We say that $(J, \Omega, P: \Omega \rightarrow X)$ is a Schottky uniformization of $X$. A simple proof of this fact was also given by L. Bers in [1] using quasiconformal mappings.

In case we can choose the above loops $\alpha_{j}$ and $\widetilde{\alpha}_{j}$ (for all $j$ ) as Euclidean circles, for some set of free generators of the Schottky group, $J$ is called a classical Schottky group. The existence of non-classical Schottky groups is well known [15] and an explicit example was given by Yamamoto [21]. A theorical construction of infinitely many non classical Schottky groups in every genus has been developed by B. Maskit and R.A. Hidalgo [13]. An open problem is to determine if every closed Riemann surface can be uniformized by a classical Schottky group.

A Schottky group $J$ is called hyperelliptic if there is a Moebius transformation $C$ of order two such that $C J C^{-1}=J$ and the group generated by $J$ and $C$ uniformizes a sphere (necessarily with $2(p+1)$ branched points of order two). Equivalently, there is a set of free generators $A_{1}, \ldots, A_{p}$ for $J$ so that $C A_{j} C=A_{j}^{-1}$ for $j=1, \ldots, p$. The hyperelliptic Schottky groups uniformize hyperelliptic Riemann surfaces and, reciprocally, every hyperelliptic Riemann surface can be uniformized by such a group [14].

In a similar way, we have the class of real Schottky groups which permits to uniformize Riemann surfaces with reflections. A Schottky group $J$ is called real if there is a reflection $\sigma$ (an anticonformal automorphism of order two of the Riemann sphere with a circle of fixed points) for which $\sigma \gamma \sigma=\gamma$, for every $\gamma \in J$. We call $\sigma$ the reflection associated to the real Schottky group $J$. Observing that then the circle $\Sigma$ of fixed points of $\sigma$ must be pre- 
served by every transformation in $J$, it follows that real Schottky groups are either Fuchsian groups or have an index two Fuchsian subgroup. It also follows that every real Schottky group is necessarily a classical Schottky group.

Theorem ([17]) Let $R$ be a closed Riemann surface admitting a reflection $\tau$. Then $R$ may be uniformized by a real Schottky group, with associated reflection $\sigma$, so that $\sigma$ is a lift of $\tau$.

A lifting problem concerning Schottky uniformizations of closed Riemann surfaces is the following. Assume we have a closed Riemann surface $X$ and a group $H$ of conformal automorphisms of it. In general, there is no Schottky uniformization of $X$ for which the group $H$ lifts. It is then natural to ask for conditions in order to have the existence of a Schottky uniformization of $X$ for which this lifting is possible. When this is the case, we say that $H$ is of Schottky type.

In [7] we have found easy necessary conditions to be satisfied by $H$ in order for it to be of Schottky type (condition (A)). These conditions are trivially satisfied by groups isomorphic to a dihedral group and for groups acting without fixed points. In [7], [8], [9] and [10] we have shown that these necessary conditions turn out to be sufficient for abelian groups, dihedral groups, the alternating groups $\mathcal{A}_{4}$ and $\mathcal{A}_{5}$ and for the symmetric group $\mathcal{S}_{4}$. In the case of groups admitting orientation-reversing transformations, we have some results for the cyclic case in [12]. Maskit's above result implies the following

Corollary 3.1 Let $R$ be a closed Riemann surface with a reflection $\tau$. Then $K(R, \tau)$ is of Schottky type.

The reason for this is as follows. Let us assume we have a closed Riemann surface $R$ and an anticonformal automorphism $\tau$ as in the hypothesis of the above corollary. As a consequence of Maskit's result, we can find a Schottky group $G$ uniformizing $R$ so that the unit circle $S^{1}$ is invariant under $G$ and such that $\theta$, the reflection on $S^{1}$, is a lifting of $\tau$. Considering that $G$ is either Fuchsian or it contains a Fuchsian group of index two, it is clear that $\theta$ normalizes the Schottky generators and the result follows.

\section{Riemann period matrices and Schottky groups}

Let us assume we have a closed Riemann surface $S$ of genus $p$ together with a subgroup $H$ of its automorphisms (maybe containing orientation reversing ones). Let us consider a symplectic basis for $R$, say $\left\{\alpha_{1}, \ldots, \alpha_{p}, \beta_{1}, \ldots, \beta_{p}\right\}$, we associate its dual basis of holomorphic one forms $\left\{w_{1}, \ldots, w_{p}\right\}$; i.e., they satisfy

$$
\int_{\alpha_{i}} w_{j}=\delta_{i j}
$$


The matrix

$$
Z=\left(\int_{\beta_{j}} w_{i}\right)
$$

is the Riemann period matrix of $R$ respect to the above basis. Each automorphism $h \in H$ acts on the first homology basis of $R$ and also in the space of harmonic one-differentials. In that way, if

$$
\rho(h)=\left(\begin{array}{cc}
A_{h} & B_{h} \\
C_{h} & D_{h}
\end{array}\right)
$$

is the induced matrix of $h$ in the above symplectic homology basis, then

$$
Z= \begin{cases}\left(A_{h}+Z C_{h}\right)^{-1}\left(B_{h}+Z D_{h}\right), & \text { if } h \text { is conformal, } \\ \left(A_{h}+\bar{Z} C_{h}\right)^{-1}\left(B_{h}+\bar{Z} D_{h}\right), & \text { if } h \text { is anticonformal. }\end{cases}
$$

Now, let us assume we have a Kleinian group $K$ (which may include anticonformal Moebius transformations) containing a Schottky group $J$ of genus $p \geq 2$ with region of discontinuity $\Omega$ (also for $K$ ) as a normal subgroup of finite index so that $\Omega / J=S$ and $H=K / J$.

The above representation $\rho$ is now defined on $K$ and descends to the above one after quotient by $J$. Let us denote by $P: \Omega \rightarrow S$ a natural holomorphic covering with $J$ as covering group. Let us fix some fundamental set of loops for $J$, say $\alpha_{1}, \ldots, \alpha_{p}, \widetilde{\alpha}_{1}, \ldots, \widetilde{\alpha}_{p}$ with a fixed orientation on each, and let $A_{1}, \ldots, A_{p}$ be a set of free generators of $J$ with respect to these loops. We also consider suitable oriented pairwise disjoint paths $\beta_{1}, \ldots, \beta_{p}$ such that $\beta_{j}$ connects equivalent points in $\alpha_{j}$ and $\widetilde{\alpha}_{j}$ and such that $\beta_{j}$ is disjoint from $\alpha_{t}$ and $\widetilde{\alpha_{t}}$ for all $t \neq j$.

Let us denote again by $\alpha_{j}$ and $\beta_{j}$ the projections to $S$ of the above respective loops, which define a symplectic basis for $S$ and, in particular, a Riemann matrix $Z$ for $S$. Since the normalizer of the projected loops $\alpha_{1}, \ldots, \alpha_{p}$ is kept invariant under the action of $H$ (because of the lifting property of $H$ by $P: \Omega \rightarrow S)$, we have that $C_{k}=0, A_{k}=\Theta(k)$, and $D_{k}=$ ${ }^{t} \Theta_{k}^{-1}$ for $k \in K$ conformal and $D_{k}=-{ }^{t} \Theta_{k}^{-1}$ for $k \in K$ anticonformal in the above matrix representation, where $\Theta: K \rightarrow \mathrm{GL}(\mathrm{p}, \mathbb{Z})$ is the isomorphism induced at the level of homology on the submodule generated by the loops $\alpha_{1}, \ldots, \alpha_{p}$. On the other hand, since $J$ is a normal subgroup of $K$, we have that each $k \in K$ induces an isomorphism $\eta(k): J \rightarrow J$, defined by $\eta(k)(j)=$ $k j k^{-1}$, for $j \in J$. We have also an isomorphism $\eta(k)^{a b}: J /[J, J] \rightarrow J /[J, J]$. It is not hard to check the following (just consider the action of $K$ on the loops $\alpha_{1}, . ., \alpha_{p}$ and their translates under $J$ ):

$$
\begin{cases}\Theta(k)={ }^{t} \eta\left(k^{-1}\right)^{a b} & k \in K \text { conformal, } \\ \Theta(k)=-{ }^{t} \eta\left(k^{-1}\right)^{a b} & k \in K \text { anticonformal. }\end{cases}
$$




\subsection{Real Schottky uniformizations case}

Let us now assume that $J$ is a real Schottky group, of genus $p \geq 2$, with associated reflection $\theta$, where $C$ denotes its circle of fixed points. In this case, each transformation of $K$ keeps invariant $C$ and commutes with $\theta$.

The surface $S$ uniformized by $J$ has a reflection induced by $\theta$ (we denote it by the same name) which commutes with each automorphism of $S$ induced by each transformation of $K$. In this case, the above procedure asserts that $\eta(\theta)^{a b}$ is the identity matrix; that is, $\Theta(\theta)=-I$. It follows that

$$
\operatorname{Re}(Z)=-\frac{1}{2} B_{\theta}
$$

We must observe that the computation of $B_{\theta}$ is simple in our case. We also have the following identities:

$$
B(\gamma)=\frac{1}{2}\left(B_{\theta} \eta(\gamma)^{a b}-{ }^{t} \eta(\gamma)^{a b} B_{\theta}\right)=\frac{1}{2}\left(B_{\theta}{ }^{t} \Theta(\gamma)^{-1}-\Theta(\gamma) B_{\theta}\right),
$$

if $\gamma \in K$ is conformal, or

$$
B(\gamma)=\frac{1}{2}\left(B_{\theta} \eta(\gamma)^{a b}+{ }^{t} \eta(\gamma)^{a b} B_{\theta}\right)=-\frac{1}{2}\left(B_{\theta}{ }^{t} \Theta(\gamma)^{-1}+\Theta(\gamma) B_{\theta}\right) .
$$

if $\gamma \in K$ is anti-conformal.

Furthermore, we obtain that for any $\gamma$ in $K$ the following holds.

$$
\Theta(\gamma) \operatorname{Im}(Z)=\operatorname{Im}(Z)^{t} \Theta(\gamma)^{-1}
$$

\section{First family: Groups of order $8 p$}

Let $\Delta$ be the unit disc and $S^{1}$ its boundary circle. For each fixed integer $p \geq 2$, we denote by $L_{1}$ the real line, $L_{2}$ the line through zero and $e^{\frac{i \pi}{2 p}}$ and $L_{3}$ a circle orthogonal to both $S^{1}$ and $L_{2}$ and disjoint from $L_{1}$. If the center of $L_{3}$ is $\lambda e^{\frac{i \pi}{2 p}}$, then the above condition is equivalent to $1<\lambda<\frac{1}{\cos \left(\frac{\pi}{2 p}\right)}$.

Let $\theta, \sigma, \tau$ and $\eta=\eta_{\lambda}$ be the reflections on $S^{1}, L_{1}, L_{2}$ and $L_{3}$, respectively. If we denote by $\widehat{K_{\lambda}}$ the group generated by the above reflections, then we have that

$$
\begin{aligned}
\widehat{K_{\lambda}}=\left\langle\theta, \sigma, \tau, \eta: \theta^{2}\right. & =\sigma^{2}=\tau^{2}=\eta^{2}=(\tau \sigma)^{2 p}=(\tau \eta)^{2} \\
& \left.=(\theta \sigma)^{2}=(\theta \tau)^{2}=(\theta \eta)^{2}=1\right\rangle .
\end{aligned}
$$

Denoting by $K_{\lambda}$ the index two subgroup generated by the reflections $\sigma, \tau$ and $\eta$, we have

$$
K_{\lambda}=\left\langle\sigma, \tau, \eta: \sigma^{2}=\tau^{2}=\eta^{2}=(\tau \sigma)^{2 p}=(\tau \eta)^{2}=1\right\rangle .
$$


Set $W=\tau \sigma$ the rotation of order $2 p, Q=W^{p}$ the antipodal map (rotation by $\pi$ fixing 0 and $\infty$ ) and $E=E_{\lambda}=\eta \tau$ (a conformal involution).

Let $J_{\lambda}$ be the smallest normal subgroup of $K_{\lambda}$ containing $Q E$. It turns out that $J_{\lambda}$ is a real and hyperelliptic Schottky group of genus $p$ with free generators $A_{1}=Q E$ and $A_{j}=W^{j} Q E W^{-j}$, where $2 \leq j \leq p-1$ (see Figure 1 for the case $p=2$ ). Since $\theta$ commutes with all elements in $K_{\lambda}$, we have that $J_{\lambda}$ is also a normal subgroup of $\widehat{K_{\lambda}}$.

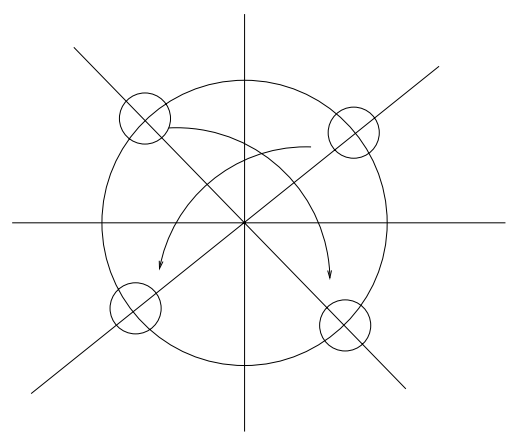

FiguRe 1.

Observe also that $A_{j}=Q \eta_{j-1} \tau_{j-1}$, where $\eta_{j}=W^{j} \eta W^{-j}$ and $\tau_{j}=\tau W^{-2 j}$. This gives us the following:

(i) $\theta A_{j} \theta=A_{j}$;

(ii) $\tau A_{1} \tau=A_{1}$;

(iii) $\tau A_{j} \tau=A_{p+2-j}^{-1}$;

(iv) $\sigma A_{j} \sigma=A_{p+1-j}^{-1}$;

(v) $\eta A_{j} \eta=A_{1}^{-1} A_{p+2-j} A_{1}$,

where the sub-indices for the $A$ 's are modulo $p$. In particular, note that (v) implies the equality

$$
\eta A_{1} \eta=A_{1} .
$$

The quotient $G_{\lambda}=K_{\lambda} / J_{\lambda}$ is generated by three elements $x, y$ and $z$ with relations:

$$
x^{2}=y^{2}=z^{2}=(y x)^{2 p}=(y z)^{2}=(y x)^{p} z y=1,
$$

that is,

$$
G_{\lambda}=\left\langle x, y: x^{2}=y^{2}=(y x)^{2 p}\right\rangle,
$$

and, in particular, $G_{\lambda}$ is isomorphic to the dihedral group of order $4 p$.

If we denote by $\Omega_{\lambda}$ the region of discontinuity of $\widehat{K_{\lambda}}$, then it is also the region of discontinuity of each of the above normal subgroups. Denoting by $H$ the group generated by $\theta$ (a normal subgroup of $K_{\lambda}$ ), we have that $\overline{\Delta_{\lambda}}=\Omega_{\lambda} / H$ is the closed disc $\bar{\Delta}$ minus a Cantor set on its boundary (the limit set of $\widehat{K_{\lambda}}$ ). 
We have that $S_{\lambda}=\overline{\Delta_{\lambda}} / J_{\lambda}$ is a compact bordered Riemann surface of algebraic genus $p$, its double is the closed Riemann surface of genus $p$ uniformized by the Schottky group $J_{\lambda}$, that is, $\Omega_{\lambda} / J_{\lambda}$. The surface $S_{\lambda}$ admits the group $G_{\lambda}$ as group of automorphisms.

Moreover, $S_{\lambda} / G_{\lambda}$ is uniformized by $\Omega_{\lambda} / \widehat{K_{\lambda}}=\overline{\Delta_{\lambda}} / K_{\lambda}$ which is a closed disc with four branched values in the border, one of order $2 p$ and three of order 2.

We have thus produced, for each genus $p \geq 2$, a one real dimension family of real Schottky groups of genus $p$ uniformizing May surfaces of genus $p$ with group of automorphisms of order $8 p$ commuting with a reflection on it.

\section{Second family: Groups of order $8(p+1)$}

Fix an integer $p \geq 2$ and denote by $L_{1}$ the real line, $L_{2}$ the line through zero and $e^{\frac{i \pi}{(p+1)}}$ and $L_{3}$ a circle orthogonal to both $S^{1}$ and $L_{1}$ and disjoint from $L_{2}$. If we denote by $\lambda$ the center of $L_{3}$, then the above condition is equivalent to $1<\lambda<\frac{1}{\cos \left(\frac{\pi}{p+1}\right)}$.

Let $\theta, \sigma, \tau$ and $\eta=\eta_{\lambda}$ be the reflections on $S^{1}, L_{1}, L_{2}$ and $L_{3}$, respectively. Let us also fix some integer $k \in\{1, \ldots, p\}$.

If we denote by $\widehat{K_{\lambda}}$ the group generated by the above reflections, then we have that

$$
\begin{aligned}
\widehat{K_{\lambda}}=\left\langle\theta, \sigma, \tau, \eta: \theta^{2}\right. & =\sigma^{2}=\tau^{2}=\eta^{2}=(\tau \sigma)^{p+1}=(\sigma \eta)^{2} \\
& \left.=(\theta \sigma)^{2}=(\theta \tau)^{2}=(\theta \eta)^{2}=1\right\rangle .
\end{aligned}
$$

Denote also by $K_{\lambda}$ the index two subgroup generated by the reflections $\sigma, \tau$ and $\eta$. We have

$$
K_{\lambda}=\left\langle\sigma, \tau, \eta: \sigma^{2}=\tau^{2}=\eta^{2}=(\tau \sigma)^{p+1}=(\sigma \eta)^{2}=1\right\rangle .
$$

Set $W=\tau \sigma$ the rotation of order $(p+1)$ and $\eta_{j}=W^{j} \eta W^{-j}$ the reflections on the translates of the circle $L_{3}$ by the rotation $W$. Let $J_{k}^{\lambda}$ be the smallest normal subgroup of $K_{\lambda}$ containing $T_{k}=\eta W^{k} \eta W^{-1}=\eta(\tau \sigma)^{k-1} \tau \eta \tau$.

The quotient $G_{k}^{\lambda}=K_{\lambda} / J_{k}^{\lambda}$ has presentation

$$
G_{k}^{\lambda}=\left\langle x, y, z: x^{2}=y^{2}=z^{2}=(x y)^{p+1}=(y z)^{2}=1, z x z=y(x y)^{r}\right\rangle,
$$

where $x, y$ and $z$ are the classes representing $\tau, \sigma$ and $\eta$, respectively and $r=p+1-k$ (see Bujalance's theorem). This is a group of order $4(p+1)$ if and only if $r^{2} \equiv 1 \bmod (p+1)$.

If we denote by $\Omega_{\lambda}$ the region of discontinuity of $\widehat{K_{\lambda}}$, then it is also the region of discontinuity of each of the above normal subgroups. Denoting 
by $H$ the group generated by $\theta$ (a normal subgroup of $\widehat{K_{\lambda}}$ ), we have that $\overline{\Delta_{\lambda}}=\Omega_{\lambda} / H$ is the closed disc $\bar{\Delta}$ minus a Cantor set on its boundary (the limit set of $\widehat{K_{\lambda}}$ ).

In general, the subgroup $J_{k}^{\lambda}$ will have torsion and, in particular, it will not be a Schottky group. For instance, if we take $p=3$ and $k=2$, then $J_{2}^{\lambda}$ contains the transformations $T_{2}$ and $\sigma T_{2}^{-1} \sigma$. But $\sigma T_{2}^{-1} \sigma T_{2}=W^{2}$ and therefore the group $J_{2}^{\lambda}$ contains torsion. More precisely, we have the following:

Proposition $6.1 J_{k}^{\lambda}$ is a real Schottky group of genus $p$ if and only if $k^{2} \equiv 1$ $\bmod (p+1)$.

Proof. Let us write down first some easy relations satisfied by the transformations $\tau, \eta_{j}, \eta, \sigma$ and $W$.

(1) $\tau \eta \tau=\eta_{1}$

(2) $\tau \eta_{j} \tau=\eta_{p+2-j}$, for $j=2, \ldots, p$;

(3) $\sigma \eta \sigma=\eta$;

(4) $\sigma \eta_{j} \sigma=\eta_{p+1-j}$, for $j=2, \ldots, p$;

(5) $W^{n} \eta_{j} W^{-n}=\eta_{j+n}$, for all $j=1, \ldots, p$.

For a fixed $k \in\{1, \ldots, p\}$, we consider the hyperbolic transformations $A_{j}=$ $\eta W^{k j} \eta W^{-j}$, for $j=1, \ldots, p$. For instance, if $k=1$, then $A_{j}=\eta \eta_{j}$ and if $k=p$, then $A_{j}=\eta W^{-2 j} \eta_{j}$. Let us denote by $\widehat{J}_{k}$ the group generated by the transformations $A_{1}, \ldots, A_{p}$. We have the following relations as consequences of (1)-(5):

(a) $\eta A_{j} \eta=\eta \eta W^{j(k-1)} \eta_{j} \eta=W^{j(k-1)} \eta_{j} \eta=\eta_{j k} W^{j(k-1)} \eta=$

$$
=\eta_{j k} W^{-j k(k-1)} \eta \eta W^{\left(k^{2}-1\right) j} \eta=A_{j k}^{-1} \eta W^{j\left(k^{2}-1\right)} \eta, \quad \text { for } j=1, \ldots, p ;
$$

(b) $\tau A_{1} \tau=\tau \eta W^{k-1} \eta_{1} \tau=\eta_{1} \tau(\tau \sigma)^{k-1} \tau \eta=\eta_{1}(\sigma \tau)^{k-1} \eta=\eta_{1} W^{-(k-1)} \eta=A_{1}^{-1}$;

(c) $\tau A_{j} \tau=\tau \eta W^{j(k-1)} \eta_{j} \tau=\eta_{1} \tau(\tau \sigma)^{j(k-1)} \tau \eta_{1-j}=\eta_{1}(\sigma \tau)^{j(k-1)} \eta_{1-j}=$

$$
\begin{aligned}
& =\eta_{1} W^{-j(k-1)} \eta_{1-j}=\eta_{1} W^{-(k-1)} \eta \eta W^{(1-j)(k-1)} \eta_{1-j}= \\
& =A_{1}^{-1} A_{1-j}, \quad \text { for } j=2, \ldots, p ;
\end{aligned}
$$

(d) $\sigma A_{j} \sigma=\sigma \eta W^{j(k-1)} \eta_{j} \sigma=\eta \sigma(\tau \sigma)^{j(k-1)} \sigma \eta_{p+1-j}=\eta(\sigma \tau)^{j(k-1)} \eta_{p+1-j}=$

$$
=\eta W^{-j(k-1)} \eta_{p+1-j}=A_{p+1-j}, \quad \text { for } j=1, \ldots, p .
$$

Since $\tau \sigma A_{j} \sigma \tau=A_{1}^{-1} A_{j+1}, A_{1}$ belongs to $J_{k}^{\lambda}$ and $J_{k}^{\lambda}$ is a normal subgroup in $K_{\lambda}$, we get inductively that $A_{2}, \ldots, A_{p}$ also belong to $J_{k}^{\lambda}$ and, in particular, $\widehat{J}_{k}<J_{k}^{\lambda}$. Part (a) says that $W^{j\left(k^{2}-1\right)}$ belongs to $J_{k}^{\lambda}$. In particular, we have that $J_{k}^{\lambda}$ has torsion if $k^{2}$ is not equivalent to $1 \bmod (p+1)$ and, in particular, $J_{k}^{\lambda}$ cannot be a Schottky group. 
On the other hand, if $k^{2} \equiv 1 \bmod (p+1)$, then $(\mathrm{a})-(\mathrm{d})$ asserts that $\widehat{J}_{k}$ is a normal subgroup of $K_{\lambda}$ containing $T_{k}=A_{1}$ and, in consequence, we must have that $\widehat{J}_{k}=J_{k}^{\lambda}$. But, $\widehat{J}_{k}$ turns out to be a Schottky group by construction (see lemma below) if we have $k^{2} \equiv 1 \bmod (p+1)$. See Figures 2 and 3 for $p=2$.

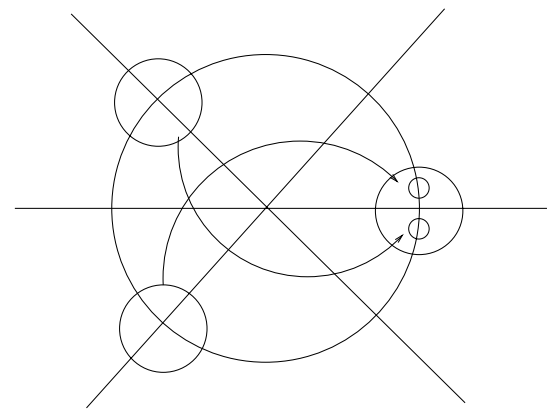

FiguRE 2: $p=2, k=2, r=1$.

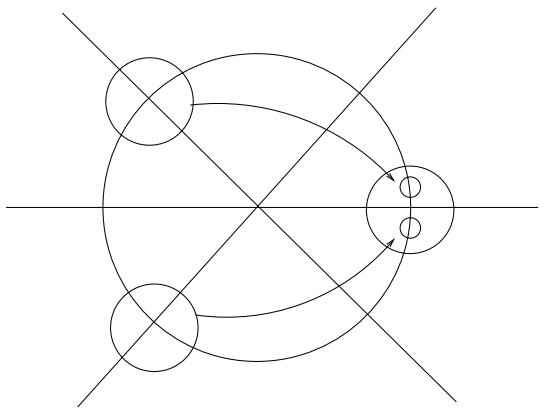

Figure 3: $p=2, k=1, r=2$.

Since the reflection $\theta$ commutes with every transformation above, we obtain also that $\widehat{J}_{k}$ is in fact a real Schottky group.

Lemma 6.2 If $k^{2} \equiv 1 \bmod (p+1)$, then $\widehat{J}_{k}$ is a Schottky group of genus $p$. Proof. Set $C_{j}=W^{j}\left(L_{3}\right)$ for each $j \in\{1, \ldots, p\}$. Note that $A_{j}\left(C_{j}\right)=$ $\eta W^{k j}\left(L_{3}\right)$ is one of the circles obtained by reflecting the circles $C_{*}$ on $L_{3}$. Furthermore, the exterior of $C_{j}$ is sent by $A_{j}$ to the interior of the image circle.

Therefore, we only need to check that $A_{r}\left(C_{r}\right) \neq A_{R}\left(C_{R}\right)$ for each pair $r \neq R$, where $r, R \in\{1, \ldots, p\}$. If we assume $r>R$ and the equality $A_{r}\left(C_{r}\right)=A_{R}\left(C_{R}\right)$, then we obtain $A_{R}^{-1} A_{r} \eta_{r}\left(A_{R}^{-1} A_{r}\right)^{-1}=\eta_{R}$. As a consequence, it follows that $\eta_{R}=\eta_{r+(r-R)(k-1)}$ and, in particular, $(r-R) k \equiv 0$ $\bmod (p+1)$.

Since we are assuming that $k^{2} \equiv 1 \bmod (p+1)$, we have that the prime factors of $(p+1)$ are either prime factors of $(k-1)$ or $(k+1)$. In particular, they cannot be prime factors of $k$. We must have that $r-R \equiv 0 \bmod (p+1)$, giving a contradiction to the fact that $0<r-R<p-1$.

As a consequence of the above we have that $J_{k}^{\lambda}$ is a real Schottky group for each $k \in\{1, \ldots, p\}$ such that $k^{2} \equiv 1 \bmod (p+1)$. We also have that $S_{k}^{\lambda}=\overline{\Delta_{\lambda}} / J_{k}^{\lambda}$ is a compact bordered Riemann surface of algebraic genus $p$, with double cover the closed Riemann surface of genus $p$ uniformized by the real Schottky group $J_{k}^{\lambda}$; that is, $\Omega_{\lambda} / J_{k}^{\lambda}$.

Moreover, the surface $S_{k}^{\lambda}$ admits the group $G_{k}^{\lambda}$ as group of automorphisms so that $S_{k}^{\lambda} / G_{k}^{\lambda}=\Omega_{\lambda} / \widehat{K_{\lambda}}=\overline{\Delta_{\lambda}} / K_{\lambda}$ is a closed disc with four branched values in the border, one of order $(p+1)$ and three of order 2 . The condition on $k$ also asserts that $G_{k}^{\lambda}$ has order $4(p+1)$ (see Bujalance's theorem). 
In this way, one gets a one real dimensional family of real Schottky uniformizations of real closed Riemann surfaces $S_{\lambda}^{d}$ of genus $p$ admitting an anticonformal involution $\theta_{\lambda}: S_{k}^{\lambda} \rightarrow S_{k}^{\lambda}$ so that $S_{k}^{\lambda} / \theta_{\lambda}$ is a bordered compact Riemann surface of algebraic genus $p$ with a group of automorphisms of order $4(p+1)$ as in Bujalance's theorem.

Remark. Let us remark that for $k=1$ and $k=p$ the Schottky group $J_{k}^{\lambda}$ is also hyperelliptic, the hyperelliptic involution is induced by the involution $C=\sigma \eta$.

\section{The principally polarized abelian varieties associated to the $8 p$-family}

In the construction of section 5, we obtained a 1-dimensional real family $J_{\lambda}$ of classical Schottky groups of genus $p \geq 2$, each invariant under a group $K_{\lambda}$ of order $8 p$. The group $K_{\lambda}$ is generated by the reflections $\theta, \sigma, \tau$ and $\eta=\eta_{\lambda}$. The Schottky group $J=J_{\lambda}$ is freely generated by transformations $A_{1}, \ldots, A_{p}$, so that:

(i) $\theta A_{j} \theta=A_{j}$;

(ii) $\tau A_{1} \tau=A_{1}$;

(iii) $\tau A_{j} \tau=A_{p+2-j}^{-1}$;

(iv) $\sigma A_{j} \sigma=A_{p+1-j}^{-1}$; and

(v) $\eta A_{j} \eta=A_{1}^{-1} A_{p+2-j} A_{1}$.

We have a natural faithful representation $\Theta: K_{\lambda} \rightarrow G L(p, \mathbb{Z})$ defined by the action on homology at the level of the alpha loops defined by the generators $A_{j}$ on the surface uniformized by $J$, given as follows.

$$
\begin{gathered}
\Theta(\theta)=-I, \\
\Theta(\tau)=-\Theta(\tau), \\
\Theta\left(\begin{array}{cccc}
-1 & 0 & \cdots & 0 \\
0 & 0 & \cdots & 1 \\
\vdots & \vdots & \vdots & \vdots \\
0 & 1 & \cdots & 0
\end{array}\right), \Theta(\sigma)=\left(\begin{array}{ccccc}
0 & 0 & \cdots & 0 & 1 \\
0 & 0 & \cdots & 1 & 0 \\
\vdots & \vdots & \vdots & \vdots & \vdots \\
1 & 0 & \cdots & 0 & 0
\end{array}\right)
\end{gathered}
$$

If we choose some beta loops to obtain a symplectic basis, we obtain a symplectic faithful representation

$$
\begin{array}{rrr}
\rho(\theta)=\left(\begin{array}{cc}
-I & B_{\theta} \\
0 & I
\end{array}\right) & \rho(\tau)=\left(\begin{array}{cc}
\Theta(\tau) & B_{\tau} \\
0 & -\Theta(\tau)
\end{array}\right) \\
\rho(\sigma)=\left(\begin{array}{cc}
\Theta(\sigma) & B_{\sigma} \\
0 & -\Theta(\sigma)
\end{array}\right) & \rho(\eta)=\left(\begin{array}{cc}
-\Theta(\tau) & B_{\eta} \\
0 & \Theta(\tau)
\end{array}\right)
\end{array}
$$


where ${ }^{t} B_{\theta}=B_{\theta},{ }^{t} B_{\tau}=B_{\tau},{ }^{t} B_{\sigma}=B_{\sigma},{ }^{t} B_{\eta}=B_{\eta}, \Theta(\tau) B_{\tau}=B_{\tau} \Theta(\tau)$, $\Theta(\sigma) B_{\sigma}=B_{\sigma} \Theta(\sigma)$, and $\Theta(\tau) B_{\eta}=B_{\tau} \Theta(\eta)$.

By construction, the group $\rho\left(K_{\lambda}\right)$ has fixed points in the Siegel space $\mathbb{H}_{p}$. Some of these fixed points are Jacobians of closed Riemann surfaces. In particular, we have family of principally polarized abelian varieties having the group $\rho(K)$ as group of automorphisms (admitting orientation reversing ones).

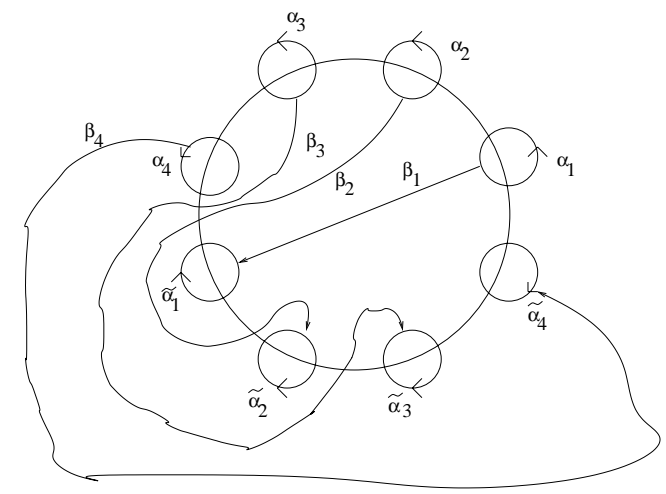

FiguRE 4.

Theorem 7.1 If we choose the beta loops in a suitable manner (as shown in Figure 4 for $p=4)$, then the locus of fixed points of $\rho\left(K_{\lambda}\right)$ in $\mathbb{H}_{p}$ is a real family of dimension $[(p+1) / 2]$ (with [] denoting integer part) given by the matrices $Z=-\frac{1}{2} B_{\theta}+Y$, where

$$
B_{\theta}=\left(\begin{array}{cccccc}
0 & 1 & 1 & \cdots & 1 & 1 \\
1 & 0 & 1 & \cdots & 1 & 1 \\
1 & 1 & 0 & \cdots & 1 & 1 \\
\vdots & \vdots & \vdots & \cdots & \vdots & \vdots \\
1 & 1 & 1 & \cdots & 0 & 1 \\
1 & 1 & 1 & \cdots & 1 & 0
\end{array}\right)
$$

and

$$
Y=a I+\sum_{k=1}^{L(p)} b_{k}\left(S^{k}+{ }^{t} S^{k}-S^{p-k}-{ }^{t} S^{p-k}\right),
$$

where $L(p)=\frac{p-2}{2}$ for $p$ even and $L(p)=\frac{p-1}{2}$ for $p$ odd,

$$
S=\left(\begin{array}{ccccc}
0 & 1 & 0 & \cdots & 0 \\
0 & 0 & 1 & \cdots & 0 \\
\vdots & \vdots & \vdots & \cdots & \vdots \\
0 & 0 & 0 & \cdots & 1 \\
0 & 0 & 0 & \cdots & 0
\end{array}\right),
$$

$I$ is the identity matrix of order $p$, and $a, b_{k}$ are real numbers so that $Y$ is positive definite. 
For instance, in genera $p=2,3,4,5,6$ these matrices $Y$ are as follows:

$$
\begin{gathered}
Y=\left(\begin{array}{rr}
a & 0 \\
0 & a
\end{array}\right) \quad Y=\left(\begin{array}{rrr}
a & b & -b \\
b & a & b \\
-b & b & a
\end{array}\right) \quad Y=\left(\begin{array}{rrrrr}
a & b & 0 & -b \\
b & a & b & 0 \\
0 & b & a & b \\
-b & 0 & b & a
\end{array}\right) \\
Y=\left(\begin{array}{rrrrr}
a & b & c & -c & -b \\
b & a & b & c & -c \\
c & b & a & b & c \\
-c & c & b & a & b \\
-b & -c & c & b & a
\end{array}\right) \quad Y=\left(\begin{array}{rrrrrr}
a & b & c & 0 & -c & -b \\
b & a & b & c & 0 & -c \\
c & b & a & b & c & 0 \\
0 & c & b & a & b & c \\
-c & 0 & c & b & a & b \\
-b & -c & 0 & c & b & a
\end{array}\right)
\end{gathered}
$$

Proof. Let $Z=X+i Y \in \mathbb{H}_{p}$ be a fixed point of $\rho\left(K_{\lambda}\right)$. It follows from (4.1) and (4.3) that then

$$
B_{\tau}=-\frac{1}{2}\left(B_{\theta} \Theta(\tau)+\Theta(\tau) B_{\theta}\right), \quad B_{\sigma}=-\frac{1}{2}\left(B_{\theta} \Theta(\sigma)+\Theta(\sigma) B_{\theta}\right),
$$

$X=-\frac{1}{2} B_{\theta}$ and $B_{\eta}=-B_{\tau}$. Using the chosen $\alpha$ and $\beta$ loops, we obtain the specific form for $B_{\theta}$ given in the statement of the theorem; then we may use (7.1) to explicitly compute the other $B$ 's and finally obtain $Y$ from (4.4), finishing the proof.

\section{The principally polarized abelian varieties associated to the $8(p+1)$-family}

In the construction of section 6 we obtained a 1-dimensional real family $J_{k}^{\lambda}$ of classical Schottky groups of genus $p \geq 2$, each invariant under a group $K_{\lambda}$ of order $8(p+1)$. The group $K_{\lambda}$ is generated by the reflections $\theta, \sigma, \tau$ and $\eta_{k}$. The Schottky group $J=J_{k}^{\lambda}$ is freely generated by transformations $A_{1}, \ldots, A_{p}$, so that:

(i) $\theta A_{j} \theta=A_{j}$,

(ii) $\tau A_{1} \tau=A_{1}^{-1}$,

(iii) $\tau A_{j} \tau=A_{1}^{-1} A_{1-j}$,

(iv) $\sigma A_{j} \sigma=A_{p+1-j}$, and

(v) $\eta_{k} A_{j} \eta_{k}=A_{k j}^{-1}$. 
The last relation is a consequence of (a) in the proof of proposition 6.1, since we are in the case $k^{2} \equiv 1(\bmod ) p+1$. In this case the natural faithful representation $\Theta: K_{\lambda} \rightarrow G L(p, \mathbb{Z})$ defined by the action on homology at the level of the alpha loops defined by the generators $A_{j}$ on the surface uniformized by $J$ is given as follows.

$$
\Theta(\theta)=-I, \Theta(\tau)=\left(\begin{array}{cccc}
1 & 0 & \cdots & 0 \\
1 & 0 & \cdots & -1 \\
\vdots & \vdots & \vdots & \vdots \\
1 & -1 & \cdots & 0
\end{array}\right), \Theta(\sigma)=\left(\begin{array}{cccc}
0 & 0 & \cdots & -1 \\
0 & 0 & \cdots & 0 \\
\vdots & \vdots & \vdots & \vdots \\
-1 & 0 & \cdots & 0
\end{array}\right)
$$

The transformation $\Theta\left(\eta_{k}\right)$ will depend on the value of $k \in\{1, \ldots, p\}$ so that $k^{2} \equiv 1 \bmod (p+1)$, but it will be a symmetric matrix since $j \equiv k j \bmod (p+1)$. In the particular cases of $k \in\{1, p\}$, we have:

$$
\Theta\left(\eta_{1}\right)=I \quad \text { and } \quad \Theta\left(\eta_{p}\right)=-\Theta(\sigma)
$$

For $p=7$, the other possible values of $k$ are $k=3$ and $k=5$; in those cases we have the following corresponding matrices.

$$
\Theta\left(\eta_{3}\right)=\left(\begin{array}{ccccccc}
0 & 0 & 1 & 0 & 0 & 0 & 0 \\
0 & 0 & 0 & 0 & 0 & 1 & 0 \\
1 & 0 & 0 & 0 & 0 & 0 & 0 \\
0 & 0 & 0 & 1 & 0 & 0 & 0 \\
0 & 0 & 0 & 0 & 0 & 0 & 1 \\
0 & 1 & 0 & 0 & 0 & 0 & 0 \\
0 & 0 & 0 & 0 & 1 & 0 & 0
\end{array}\right), \quad \Theta\left(\eta_{5}\right)=\left(\begin{array}{ccccccc}
0 & 0 & 0 & 0 & 1 & 0 & 0 \\
0 & 1 & 0 & 0 & 0 & 0 & 0 \\
0 & 0 & 0 & 0 & 0 & 0 & 1 \\
0 & 0 & 0 & 1 & 0 & 0 & 0 \\
1 & 0 & 0 & 0 & 0 & 0 & 0 \\
0 & 0 & 0 & 0 & 0 & 1 & 0 \\
0 & 0 & 1 & 0 & 0 & 0 & 0
\end{array}\right)
$$

If we choose some beta loops to obtain a symplectic basis, we obtain a symplectic faithful representation $\rho: K_{\lambda} \rightarrow \widetilde{\operatorname{Sp}_{2 p}(\mathbb{Z})}$ :

$$
\begin{array}{cc}
\rho(\theta)=\left(\begin{array}{cc}
-I & B_{\theta} \\
0 & I
\end{array}\right) & \rho(\tau)=\left(\begin{array}{cc}
\Theta(\tau) & B_{\tau} \\
0 & -{ }^{t} \Theta(\tau)
\end{array}\right) \\
\rho(\sigma)=\left(\begin{array}{cc}
\Theta(\sigma) & B_{\sigma} \\
0 & -\Theta(\sigma)
\end{array}\right) & \rho\left(\eta_{k}\right)=\left(\begin{array}{cc}
\Theta\left(\eta_{k}\right) & B_{\eta_{k}} \\
0 & -\Theta\left(\eta_{k}\right)
\end{array}\right)
\end{array}
$$

where ${ }^{t} B_{\theta}=B_{\theta},{ }^{t} B_{\tau}=B_{\tau},{ }^{t} B_{\sigma}=B_{\sigma},{ }^{t} B_{\eta}=B_{\eta}$,

$$
\Theta(\tau) B_{\tau}=B_{\tau} \Theta(\tau)={ }^{t} B_{\tau}{ }^{t} \Theta(\tau), \quad \Theta(\sigma) B_{\sigma}=B_{\sigma} \Theta(\sigma)
$$

and

$$
\Theta\left(\eta_{k}\right) B_{\eta_{k}}=B_{\eta_{k}} \Theta\left(\eta_{k}\right)={ }^{t} B_{\eta_{k}} \Theta\left(\eta_{k}\right) .
$$


For $k$ in $\{1, p\}$, we now fix particular $\beta$ loops as in Figures 5 and 6 respectively (for $p=3$ ).

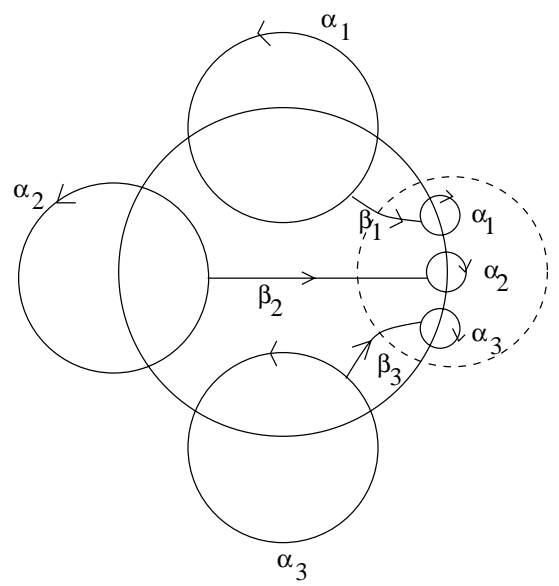

FigURE 5.

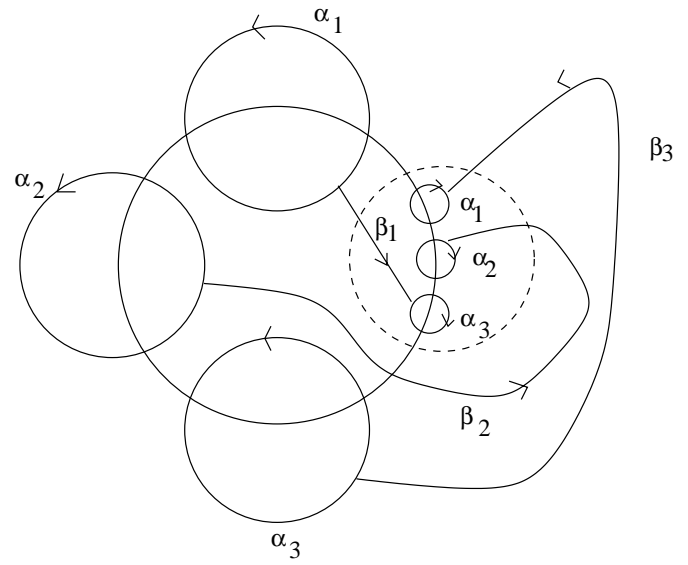

FIGURE 6.

With these choices we may find explicit matrices $B_{\theta}$, as follows.

$$
\left\{\begin{array}{l}
B_{\theta}=0 \\
B_{\theta}=\left(\begin{array}{cccccc}
0 & 1 & 1 & \cdots & 1 & 1 \\
1 & 0 & 1 & \cdots & 1 & 1 \\
1 & 1 & 0 & \cdots & 1 & 1 \\
\vdots & \vdots & \vdots & \cdots & \vdots & \vdots \\
1 & 1 & 1 & \cdots & 0 & 1 \\
1 & 1 & 1 & \cdots & 1 & 0
\end{array}\right) \quad \text { if } k=p .
\end{array}\right.
$$


Using the same arguments as before, we have now the following.

Theorem 8.1 For $k$ in $\{1, p\}$, the locus of fixed points of $\rho\left(K_{\lambda}\right)$ in $\mathbb{H}_{p}$ is a $d_{p}$-real dimensional subvariety given by the matrices $Z=-\frac{1}{2} B_{\theta}+i Y$, where $B_{\theta}$ is given above for each case, and the imaginary part $Y$ is of the following form (where $S$ is the matrix given by (7.2)):

(1) If $p=2$, then $Y^{-1}=a_{1}\left(\begin{array}{cc}-2 & 1 \\ 1 & -2\end{array}\right)$;

(2) If $p=3$, then $Y^{-1}=a_{1} I+a_{2}\left(S+{ }^{t} S\right)-\frac{a_{1}+a_{2}}{2}\left(S^{2}+{ }^{t} S^{2}\right)$;

(3) If $p=4$, then $Y^{-1}=a_{1} I+a_{2}\left(S+{ }^{t} S\right)-\left(\frac{a_{1}}{2}+a_{2}\right)\left(S^{2}+{ }^{t} S^{2}+S^{3}+{ }^{t} S^{3}\right)$;

(4) If $p=2 r+1 \geq 5$, then

$$
\begin{aligned}
Y^{-1}= & \sum_{j=0}^{r} a_{1+j}\left(S^{j}+{ }^{t} S^{j}\right)-2\left(\sum_{m=1}^{r+1} a_{m}\right)\left(S^{r+1}+{ }^{t} S^{r+1}\right) \\
& +\sum_{j=1}^{r-1} a_{r+2-j}\left(S^{r+1+j}+{ }^{t} S^{r+1+j}\right) ;
\end{aligned}
$$

(5) If $p=2 r \geq 6$, then

$$
\begin{aligned}
Y^{-1}= & \sum_{j=0}^{r-1} a_{1+j}\left(S^{j}+{ }^{t} S^{j}\right)-\left(\sum_{m=1}^{r} a_{m}\right)\left(S^{r}+{ }^{t} S^{r}+S^{r+1}+{ }^{t} S^{r+1}\right) \\
& +\sum_{j=1}^{r-2} a_{r+1-j}\left(S^{r+1+j}+{ }^{t} S^{r+1+j}\right),
\end{aligned}
$$

where the real numbers $a_{j}$ are so that $Y$ is positive definite and

$$
d_{p}= \begin{cases}\frac{p}{2} & \text { if } p \text { is even } \\ \frac{p+1}{2} & \text { if } p \text { is odd } .\end{cases}
$$

Remark. The above theorem is essentially the result of computing the fixed points in $\mathbb{H}_{p}$ for the generators $\rho(\theta), \rho(\tau)$ and $\rho(\sigma)$. These are the only transformations we need to care for in the case $k$ in $\{1, p\}$ (by (8.2)). But for the possible cases that $k \notin\{1, p\}$ (and $k^{2} \equiv 1$ (mod) $p+1$ ) we need to consider the extra transformation $\rho\left(\eta_{k}\right)$. We will now exemplify with the case $p=7$, where the possibilities are $k=3$ and $k=5$. 


\subsection{Case $p=7$}

If we choose the beta loops as shown in Figure 7 for $k=3$ and in Figure 8 for $k=5$,

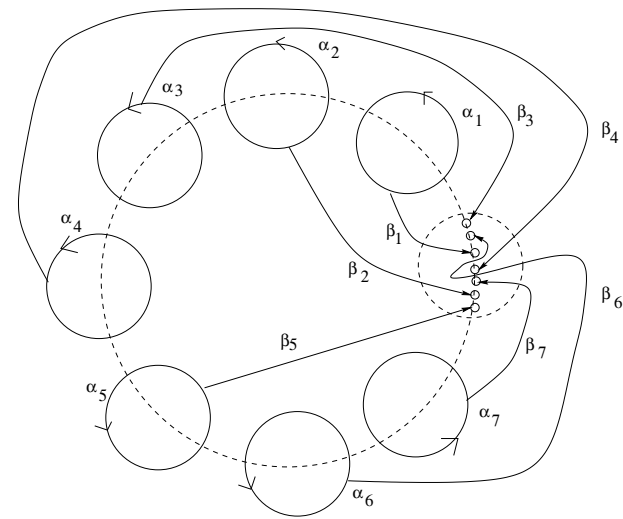

Figure 7.

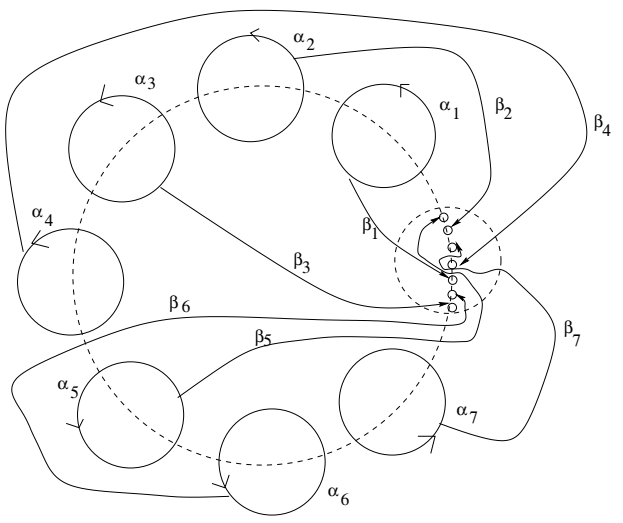

FigURE 8.

then

$$
B_{\theta}=\left\{\begin{array}{ccccccc}
0 & 0 & 1 & 0 & 0 & 1 & 0 \\
0 & 0 & 1 & 1 & 0 & 1 & 1 \\
1 & 1 & 0 & 0 & 0 & 0 & 0 \\
0 & 1 & 0 & 0 & 0 & -1 & 0 \\
0 & 0 & 0 & 0 & 0 & 1 & 1 \\
1 & 1 & 0 & -1 & 1 & 0 & 0 \\
0 & 1 & 0 & 0 & 1 & 0 & 0
\end{array}\right) \quad \text { if } k=3
$$

As for $Y$, in both cases we obtain the extra relation $a_{4}=a_{2}$ in (4) of theorem 8.1, giving a three-dimensional locus for the family of $Z$ 's fixed by the group.

Remark. Note that the Accola-Machlachlan surfaces are May surfaces with $k=p$ and the symmetry $s_{3}$ mentioned in [2], and that their period matrices, as computed in [4], coincide with those derived from our computations. 


\section{References}

[1] Bers, L.: Automorphic forms for Schottky groups. Advances in Math. 16 (1975), 332-361.

[2] Broughton, S. A., Bujalance, E., Costa, A. F., Gamboa, J. M. and GromadzKi, G.: Symmetries of Accola-Maclachlan and Kulkarni surfaces: Proc. Amer. Math. Soc. 127 (1999), 673-646.

[3] Bujalance, E.: On compact Klein surfaces with a special automorphism group. Ann. Acad. Sci. Fenn. Math. 22 (1997), 15-20.

[4] Bujalance, E., Costa, A. F., Gamboa, J. M. and Riera, G.: Period matrices of Accola-Maclachlan and Kulkarni surfaces. Ann. Acad. Sci. Fenn. Math. 25 (2000), 161-177.

[5] Chuckrow, V.: On Schottky groups with applications to kleinian groups. Ann. of Math. (2) 88 (1968), 47-61.

[6] GonzÁlez-Aguilera, V. And Rodríguez, R. E.: Families of irreducible principally polarized abelian varieties isomorphic to a product of elliptic curves. Proc. Amer. Math. Soc. 128 (2000), no. 3, 629-636.

[7] Hidalgo, R. A.: On Schottky groups with automorphisms. Ann. Acad. Sci. Fenn. Ser. A I Math. 19 (1994), 259-289.

[8] Hidalgo, R. A.: Schottky uniformizations of closed Riemann surfaces with abelian groups of conformal automorphisms. Glasgow Math. J. 36 (1994), $17-32$.

[9] Hidalgo, R. A.: Dihedral groups are of Schottky type. Proyecciones 18 (1999), 23-48.

[10] Hidalgo, R. A.: $\mathcal{A}_{4}, \mathcal{A}_{5}, \mathcal{S}_{4}$ and $\mathcal{S}_{5}$ of Schottky type. Rev. Mat. Complut. 15 (2002), no. 1, 11-29.

[11] Hidalgo, R. A.: A note on the $12(g-1)$ bound. C. R. Math. Rep. Acad. Sci. Canada 18 (1996), 39-42.

[12] Hidalgo, R. A. And Costa, A. F.: Anticonformal automorphisms and Schottky coverings. Ann. Acad. Sci. Fenn. Math. 26 (2001), 489-508.

[13] Hidalgo, R. A. And Maskit, B.: On neoclassical Schottky groups. To appear in Trans. Amer. Math. Soc.

[14] Keen, L.: On hyperelliptic Schottky groups. Ann. Acad. Sci. Fenn. Ser. A.I. Math. 5 (1980), 165-174.

[15] Marden, A.: Schottky groups and circles. In Contributions to analysis (a collection of papers dedicated to Lipman Bers), 273-278. Academic Press, New York, 1974.

[16] Maskit, B.: A characterization of Schottky groups. J. Analyse Math. 19 (1967), 227-230.

[17] Maskit, B.: Remarks on $m$-symmetric Riemann surfaces. In Lipa's legacy (New York, 1995), 433-445. Contemp. Math. 211. Amer. Math. Soc., Providence, RI, 1997. 
[18] May, C. L.: Automorphisms of compact Klein surfaces with boundary. Pacific J. Math. 59 (1975), 199-210.

[19] Rodríguez, R. E. And GonzÁlez-Aguilera, V.: Fermat's quartic curve, Klein's curve and the tetrahedron. In Extremal Riemann surfaces (San Francisco, CA, 1995), 43-62. Contemp. Math. 201. Amer. Math. Soc., Providence, RI, 1997.

[20] Unifweb: A Package for Studying Riemann Surfaces with Symmetry by Carlos O'Ryan Lira (coryan@mat.puc.cl). http://www.geom. umn.edu/apps/unifweb/start.html

[21] Yамамото, H.: An example of a nonclassical Schottky group. Duke Math. J. 63 (1991), no. 1, 193-197.

Recibido: 12 de diciembre de 2001

Revisado: 18 de noviembre de 2002

Rubén A. Hidalgo

Departamento de Matemática Universidad Técnica Federico Santa María

Valparaíso, Chile rhidalgo@mat.utfsm.cl

Rubí E. Rodríguez Departamento de Matemáticas Pontificia Universidad Católica de Chile Casilla 306-22, Santiago, Chile rubi@mat.puc.cl

Partially supported by grants Fondecyt 1030252, 1030373 y UTFSM 12.03.21, Fondecyt 1030595 and the Presidential Chair on Geometry. 